\title{
Weak-field limit of Kaluza-Klein models with spherically symmetric static scalar field: observational constraints
}

\author{
Alexander Zhuk ${ }^{1,2}$, Alexey Chopovsky ${ }^{2, a}$ (1), Seyed Hossein Fakhr ${ }^{2}$, Valerii Shulga ${ }^{1,3}$, Han Wei $^{1}$ \\ ${ }^{1}$ The International Center of Future Science of the Jilin University, 2699 Qianjin St., Changchun City 130012, China \\ ${ }^{2}$ Astronomical Observatory, Odessa National University, Dvoryanskaya st. 2, Odessa 65082, Ukraine \\ ${ }^{3}$ Institut of Radio Astronomy of National Academy of Sciences of Ukraine, 4 Mystetstv str., Kharkov 61002, Ukraine
}

Received: 9 June 2017 / Accepted: 17 October 2017 / Published online: 30 October 2017

(C) The Author(s) 2017. This article is an open access publication

\begin{abstract}
In a multidimensional Kaluza-Klein model with Ricci-flat internal space, we study the gravitational field in the weak-field limit. This field is created by two coupled sources. First, this is a point-like massive body which has a dust-like equation of state in the external space and an arbitrary parameter $\Omega$ of equation of state in the internal space. The second source is a static spherically symmetric massive scalar field centered at the origin where the point-like massive body is. The found perturbed metric coefficients are used to calculate the parameterized post-Newtonian (PPN) parameter $\gamma$. We define under which conditions $\gamma$ can be very close to unity in accordance with the relativistic gravitational tests in the solar system. This can take place for both massive or massless scalar fields. For example, to have $\gamma \approx 1$ in the solar system, the mass of scalar field should be $\mu \gtrsim$ $5.05 \times 10^{-49} \mathrm{~g} \sim 2.83 \times 10^{-16} \mathrm{eV}$. In all cases, we arrive at the same conclusion that to be in agreement with the relativistic gravitational tests, the gravitating mass should have tension: $\Omega=-1 / 2$.
\end{abstract}

\section{Introduction}

Any physical theory is viable only when it does not contradict the experimental data. Theories of gravity are not an exception to this rule. It is well known that general relativity (GR) successfully passed the test by the gravitational experiments, e.g. the relativistic gravitational tests in the solar system: the deflection of light, the time delay of radar echoes and the perihelion precession of Mercury. Consequently, any modified theories of gravity must satisfy these observations with an accuracy not less than GR. Multidimensional Kaluza-Klein (KK) models are among such modified theories of gravity.

\footnotetext{
a e-mail: a.chopovsky@yandex.ru
}

Therefore, in Refs. [1-3], we investigated this problem for multidimensional KK models with compact Ricci-flat (or toroidal, as a particular example) internal spaces. We considered the post-Newtonian gravitational field generated by discrete massive sources with negligible (in comparison to c) velocities. We assumed that massive bodies possess zero pressure in the external (our) space. Here, the pressure is understood as a characteristic of a gravitating body. It is well known that the pressure inside the non-relativistic astrophysical bodies (such as our Sun) is much less than the energy density. Therefore, we can neglect it. This is a natural assumption in general relativity [4] accepted for calculation of the parameterized post-Newtonian (PPN) parameters [5]. It is natural to assume that in the internal space the gravitating mass also has a dust-like equation of state. However, since we do not know the equation of state in the internal space, we assumed for generality some non-zero parameter $\Omega$ in this equation. Next, for such a KK model we investigated the PPN parameter $\gamma$. This parameter is well defined from the Shapiro time-delay experiment: $\gamma=1+(2.1 \pm 2.3) \times 10^{-5}$ [6-8]. To our surprise, in the case of KK models with Ricciflat internal spaces, we found that to get $\gamma$ close to unity, the equation of state parameter $\Omega$ should be very close to $-1 / 2$. Strictly speaking, to have $\gamma=1$ (as in general relativity), $\Omega$ should be exactly equal to $-1 / 2$. This value is well known for black strings and black branes [9-14]. For KK models with Ricci-flat internal spaces, this result does not depend on the size of the extra dimensions [15]. ${ }^{1}$

\footnotetext{
${ }^{1}$ It is a common knowledge that $\mathrm{KK}$ theory reduces to general relativity if the compact radii go to zero. Really, the compactness and smallness of the internal spaces is a necessary condition for KK models to be in agreement with the observations. However, this is not a sufficient condition. Since the variations of the volume of internal spaces contribute to the perturbations of the metric, then this results in the fifth force/radions. Such a fifth force contradicts the observations. In the case of background Ricci-flat internal spaces, radions are massless,
} 
Since up to now there is no a physically motivated explanation of the origin of such an essentially non-zero value of the parameter $\Omega$, we are asking about the possibility of constructing the KK models, for which, on the one hand, the PPN parameter $\gamma$ will satisfy the experimental constraints (i.e. will be very close to 1 ), and, on the other hand, the parameter $\Omega$ will be 0 . This is the main aim of the present paper. To achieve this goal, we include into consideration a static spherically symmetric massive scalar field coupled with the gravitating body. Unfortunately, we demonstrate that to have the parameter $\gamma \approx 1$ in the considered model, we should still demand $\Omega=-1 / 2$.

The paper is structured as follows. In Sect. 2, we describe the model and present basic equations. In Sect. 3, we calculate the perturbed metric coefficients. This coefficients enable us to estimate the PPN parameter $\gamma$ in Sect. 4. The main results are briefly summarized in the concluding Sect. 5 .

\section{The model and basic equations}

The background spacetime is supposed to have a $(1+D)=$ $(1+3+d)$-dimensional block-diagonal metrics of the form

$\hat{g}=\hat{g}_{M N} \mathrm{~d} x^{M} \otimes \mathrm{d} x^{N}=\eta_{\mu \nu} \mathrm{d} x^{\mu} \otimes \mathrm{d} x^{\nu}+\hat{g}_{m n} \mathrm{~d} x^{m} \otimes \mathrm{d} x^{n}$,

where $\eta_{\mu \nu}=\operatorname{diag}(+1,-1,-1,-1)$ is Minkowski metric of the visible (external) 4-dimensional spacetime, and $\hat{g}_{m n}$ is Ricci-flat metric of the compact $d$-dimensional extra (internal) subspace. Hereafter, capital Latin indices run from 0 to $D$, Greek indices run from 0 to 3, small Latin ones run from 4 to $D=3+d$; index 0 is reserved for the time coordinate; hats denote background/unperturbed quantities. We also suppose that coordinates $x^{\mu}, x^{m}$ have dimension of length. Therefore, the metric coefficients are dimensionless.

Footnote 1 continued

and this property does not depend on the size of the internal space. If the gravitating masses are the only matter sources perturbing the background geometry, to eliminate such massless radions (i.e. to eliminate the contributions of the fluctuations of the volume of internal spaces to the metric perturbations) the gravitating masses should have tension $\Omega=-1 / 2$ in the internal space. This is the black branes/strings case. These models satisfy the gravitational tests in the solar system at the same level of accuracy as general relativity. In the case of curved internal spaces we have a different picture. Here, we can also eliminate radions if $\Omega=-1 / 2$. However, for such models radions are massive with masses inversely proportional to the volume of the internal space, and the contributions of the fifth force have a Yukawa type form. Therefore, for an arbitrary value of $\Omega$ such a KK theory reduces to general relativity in the limit of large masses (i.e. small internal space volume) where the Yukawa contributions are exponentially small. These aspects of KK models are discussed in detail in Refs. [15,16].
The background spacetime is perturbed by a matter source. In the weak-field limit, the perturbed metric coefficients

$g_{M N} \approx \hat{g}_{M N}+h_{M N}, \quad\left|h_{M N}\right| \ll 1$

We consider a matter source consisting of two components. The first component is a compact body of mass $m$ and with mass density $\rho$. We assume that this compact body corresponds to ordinary astrophysical objects. It is well known that the pressure inside these objects (e.g., inside our Sun) is much less than the energy density. Therefore, we can neglect it. However, we do not know the pressure of these bodies in the internal space. So, we keep it and $\Omega$ is the equation of state parameter in the internal space. We should note that the corresponding pressure is not connected with motion of gravitating masses, i.e. $\Omega$ is the parameter of a body. The value of $\Omega$ should be restricted from observations. Since we are going to define the parameterized post-Newtonian (PPN) parameter $\gamma$, it is sufficient in this case to calculate perturbations $h_{M N}$ (originating with the gravitating mass) up to $O\left(1 / c^{2}\right)$ [1]. To do it, we need to keep the energy-momentum tensor (EMT) components up to $O\left(c^{2}\right)$ terms only, and for the non-zero components we get [16]

$$
\begin{aligned}
\mathrm{T}_{00} & \approx \rho c^{2}, \quad \mathrm{~T}_{m n} \approx-\Omega \rho c^{2} \hat{g}_{m n}, \\
\mathrm{~T} & \approx \mathrm{T}_{M N} \hat{g}^{M N}=\rho c^{2}(1-\Omega d) .
\end{aligned}
$$

Hereinafter, the energy-momentum tensor related to the gravitating body will be denoted by sans typeface.

The conservation equation for this tensor takes the form $\nabla_{M} \mathrm{~T}_{N}^{M}=\hat{\nabla}_{M} \mathrm{~T}_{N}^{M}+o\left(c^{2}\right)=0$ where $o\left(c^{2}\right)$ denotes terms which are proportional to $c^{n}$ with $n<2$. We should drop such terms within the adopted accuracy. Then the gravitating body is static: ${ }^{2} \hat{\nabla}_{M} \mathrm{~T}^{M 0}=\partial_{0} \mathrm{~T}_{0}^{0}=c^{2} \partial_{0} \rho=0$ and is uniformly distributed/smeared over the internal space: $\hat{\nabla}_{M} \mathrm{~T}_{n}^{M}=\hat{\nabla}_{m} \mathrm{~T}_{n}^{m}=-\Omega c^{2} \partial_{n} \rho=0$ unless $\Omega=0$. In what follows, we will assume that the gravitating body is localized at the origin in the external space and is smeared over the internal space: $\rho=m \delta(\mathbf{r}) / V_{d}$, where $V_{d}$ is the volume of the unperturbed internal space and $\mathbf{r}=$ $\left(x^{1}, x^{2}, x^{3}\right)$. Then, within the adopted accuracy, both matter source components satisfy the conservation equations separately.

The second material component is a static spherically symmetric scalar field $\phi$ with mass $\mu$ centered at the origin (see the footnote 3 ). We assume that this scalar field is in its ground state in the internal space (there is no dependence on the extra spatial coordinates $x^{m}$ ). This field satisfies the

\footnotetext{
2 We consider only one gravitating mass placed at the origin of a reference frame. That is, in such a comoving frame, we disregard the spatial velocity of a body. Such a simplification does not affect the main results of our paper.
} 
linearized Klein-Gordon equation $\partial_{r}^{2} \phi+(2 / r) \partial_{r} \phi-\mu^{2} \phi+$ $O(h \phi)=0$ with the following solution: ${ }^{3}$

$\phi(r)=\mathcal{C} e^{-\mu r} / r$.

Here, the mass $\mu$ has dimension length ${ }^{-1}, \mathcal{C}$ is some dimensional constant and $r \equiv \sqrt{\left(x^{1}\right)^{2}+\left(x^{2}\right)^{2}+\left(x^{3}\right)^{2}}$.

The EMT (which we denote with fraktur) of the scalar field reads

$\mathfrak{T}_{M N}=\partial_{M} \phi \partial_{N} \phi-\frac{1}{2} \hat{g}_{M N}\left[\partial_{L} \phi \partial^{L} \phi-\mu^{2} \phi^{2}\right]+O\left(h \phi^{2}\right)$,

$\mathfrak{T}=-\frac{d+2}{2} \partial^{L} \phi \partial_{L} \phi+\frac{1}{2}(d+4) \mu^{2} \phi^{2}+O\left(h \phi^{2}\right)$.

It can easily be realized that terms $O\left(h \phi^{2}\right)$ result in corrections of second order for the metric coefficients. Therefore, we should drop such terms within the adopted accuracy.

Taking into account the explicit form of $\phi(2.4)$, and introducing the spherical coordinates in the external space, we find that $\partial_{L} \phi \partial^{L} \phi=\hat{g}^{r r}\left(\partial_{r} \phi\right)^{2}=-\phi^{2}(\mu r+1)^{2} / r^{2}$, where $\hat{g}^{r r}=-1$. Then the non-zero components of $\mathfrak{T}_{M N}$ take the form

$$
\begin{aligned}
& \mathfrak{T}_{00} \approx \frac{\phi^{2}}{r^{2}}\left(\mu^{2} r^{2}+\mu r+\frac{1}{2}\right), \\
& \mathfrak{T}_{\bar{\mu} \bar{\mu}} \approx\left(\partial_{r} \phi\right)^{2}\left(\partial_{\bar{\mu}} r\right)^{2}-\mathfrak{T}_{00}, \quad\left(\partial_{\bar{\mu}} r\right)^{2}=\frac{\left(x^{\bar{\mu}}\right)^{2}}{r^{2}}, \\
& \mathfrak{T}_{\bar{\mu} \bar{\nu} \bar{\nu}} \approx\left(\partial_{\bar{\mu}} \phi\right)\left(\partial_{\bar{\nu}} \phi\right)=\frac{x^{\bar{\mu}} x^{\bar{\nu}}}{r^{2}}\left(\partial_{r} \phi\right)^{2}, \quad \bar{\mu} \neq \bar{\nu}, \\
& \mathfrak{T}_{m n} \approx \hat{g}_{m n} \mathfrak{T}_{00}=\frac{\phi^{2}}{r^{2}}\left(\mu^{2} r^{2}+\mu r+\frac{1}{2}\right) \hat{g}_{m n} .
\end{aligned}
$$

Hereinafter, $\bar{\mu}, \bar{v}=1,2,3$ denote external space components. The trace $\mathfrak{T}$, after substitution of the expression for $\partial_{L} \phi \partial^{L} \phi$ into (3), immediately takes the form

$\mathfrak{T} \approx \frac{\phi^{2}}{2 r^{2}}\left[(d+2)(\mu r+1)^{2}+(d+4) \mu^{2} r^{2}\right]$.

Both matter components result in the spacetime perturbations (2.2) where $h_{M N}$ can be found from the linearized

\footnotetext{
3 To provide a condition for the gravitating massive body and scalar field to stay at the same place (i.e. to be coupled), we can introduce an interacting term $\sim \rho \phi$ into action. Since $\rho(\mathbf{r}) \sim \delta(\mathbf{r})$, this results in a delta function in the rhs of the Klein-Gordon equation. Then the solution (2.4) is valid in any point of the space, and the integration constant $\mathcal{C}$ is defined by a coupling constant. Moreover, the terms $\sim \rho \phi$ will appear also in the EMT. They describe the energy of interaction of a massive body with scalar fields localized at other massive bodies. As we can see from (2.4), such fields are exponentially suppressed for sufficiently large distances. Therefore, we will drop such interaction terms in the EMT. In other words, we consider a one-body problem.
}

Einstein equation:

$\delta R_{M N}=\kappa\left[T_{M N}-\frac{1}{d+2} T \hat{g}_{M N}\right] \equiv S_{M N}$,

where

$T_{M N}=\mathrm{T}_{M N}+\mathfrak{T}_{M N}$

is the total energy-momentum tensor and

$$
\begin{aligned}
\delta R_{M N} \approx & \frac{1}{2}\left[-\hat{\nabla}_{L} \hat{\nabla}^{L} h_{M N}+\hat{\nabla}_{L} \hat{\nabla}_{M} h_{N}^{L}\right. \\
& \left.+\hat{\nabla}_{L} \hat{\nabla}_{N} h_{M}^{L}-\hat{\nabla}_{N} \hat{\nabla}_{M} h_{L}^{L}\right]
\end{aligned}
$$

is the perturbation of the Ricci tensor up to linear (with respect to $h_{M N}$ ) terms (see Eqs. (A.5)-(A.6) in [2]). The prefactor $\kappa$ stands for $2 S_{D} G_{(D+1)} / c^{4}$, with $S_{D}$ being the total $D$-dimensional solid angle and $G_{(D+1)}$ being the constant of gravitational interaction in $(D+1)$-dimensional spacetime. It is worth noting that the combination $\kappa \phi^{2}$ is dimensionless. Since Eq. (2.12) is linear with respect to $h_{M N}$, the two material components $\mathrm{T}_{M N}$ and $\mathfrak{T}_{M N}$ perturb the background geometry independently in the considered approximation. Therefore, we can split $h_{M N}$ into the two contributions:

$h_{M N}=\mathrm{h}_{M N}+\mathfrak{h}_{M N}$,

where $\mathrm{h}_{M N}$ is engendered by

$\mathrm{S}_{M N} \equiv \kappa\left[\mathrm{T}_{M N}-\mathrm{T} \hat{g}_{M N} /(d+2)\right]$

while $\mathfrak{h}_{M N}$ is produced by

$\mathfrak{S}_{M N}=\kappa\left[\mathfrak{T}_{M N}-\mathfrak{T} \hat{g}_{M N} /(d+2)\right]$,

respectively.

It can easily be recognized that, for the mass density $\rho=m \delta(\mathbf{r}) / V_{d}$ and the scalar field of the form (2.4), the combination $S_{M N}$ is static ( $\left.\partial_{0} S_{M N}=0\right)$, smeared over the internal space $\left(\hat{\nabla}_{m} S_{M N}=0\right)$ as well as spherically symmetric in the external space $\left(S_{M N}\left(\mathbf{r}, x^{m}\right)=S_{M N}\left(r, x^{m}\right)\right)$. Therefore, the corresponding metric perturbation $h_{M N}$ must possess the same properties, that is, $h_{M N}\left(x^{M}\right)=h_{M N}\left(r, x^{m}\right)$. Moreover, it is natural to suppose that static and smeared matter sources preserve the block-diagonal structure of the spacetime:

$h=\sum_{\mu=0}^{3} h_{\mu \mu} \mathrm{d} x^{\mu} \otimes \mathrm{d} x^{\mu}+h_{m n} \mathrm{~d} x^{m} \otimes \mathrm{d} x^{n}$.

Further, since $S_{\mu \nu}(\mu, v=0,1,2,3)$ does not depend on the internal coordinates, $h_{\mu \nu}$ also must not depend on $x^{m}$ 
$\left(\partial_{m} h_{\mu \nu}=0, m=4, \ldots, D=3+d\right)$. Additionally, for the given matter sources, the EMT components $T_{m n}$ may depend on $x^{m}$ via $\hat{g}_{m n}$ only. Therefore, we may suppose that $h_{m n}$ have the form $h_{m n}\left(r, x^{m}\right)=\xi(r) \hat{g}_{m n}\left(x^{m}\right)$ (i.e. the metric on the internal space experiences a conformal perturbation). We will see below that such prescriptions for the metric perturbations $h_{M N}$ are in full agreement with the linearized Einstein equations.

Similar to the splitting (2.13), we can distinguish in $h_{m n}\left(r, x^{m}\right)=\xi(r) \hat{g}_{m n}$ contributions from different matter sources:

$h_{m n}=\mathrm{h}_{m n}+\mathfrak{h}_{m n} \equiv \xi_{f}(r) \hat{g}_{m n}+\xi_{m}(r) \hat{g}_{m n}$.

Obviously, $\xi_{f}+\xi_{m}=\xi$.

Taking all this into account, we can simplify (2.14) significantly:

$$
\begin{aligned}
\delta R_{00} \approx & \frac{1}{2} \triangle_{3} h_{00}(r), \\
\delta R_{\bar{\mu} \bar{\mu}} \approx & \frac{1}{2}\left[\triangle_{3} h_{\bar{\mu} \bar{\mu}}-2 \partial_{\bar{\mu}}^{2} h_{\bar{\mu} \bar{\mu}}\right. \\
& \left.-\partial_{\bar{\mu}}^{2}\left(h_{00}-\sum_{\bar{\lambda}} h_{\bar{\lambda} \bar{\lambda}}+\xi d\right)\right], \\
\delta R_{\bar{\mu} \bar{\nu}} \approx & -\frac{1}{2}\left[\partial_{\bar{\mu}} \partial_{\bar{\nu}} h_{\bar{\nu} \bar{\nu}}+\partial_{\bar{\mu}} \partial_{\bar{\nu}} h_{\bar{\mu} \bar{\mu}}\right] \\
& \left.+\partial_{\bar{\mu}} \partial_{\bar{\nu}}\left(h_{00}-\sum_{\bar{\lambda}} h_{\bar{\lambda} \bar{\lambda}}+\xi d\right)\right], \quad \bar{\mu} \neq \bar{v}, \\
\delta R_{m n} \approx & \frac{1}{2} \hat{g}_{m n} \triangle_{3} \xi,
\end{aligned}
$$

where $\triangle_{3} \equiv \sum_{\bar{\mu}=1}^{3} \partial_{\bar{\mu}}^{2}$.

Now, let us calculate $S_{M N}=\mathrm{S}_{M N}+\mathfrak{S}_{M N}$. Since $\mathrm{S}_{M N}$ (defined by (2.16)) is already known (see Eq. (2.3)), we need to find $\mathfrak{S}_{M N}$ only:

$$
\begin{aligned}
\mathfrak{S}_{00} & \equiv \kappa\left[\mathfrak{T}_{00}-\frac{1}{d+2} \mathfrak{T} \hat{g}_{00}\right]=-\kappa \frac{\mu^{2} \phi^{2}}{d+2}, \\
\mathfrak{S}_{\bar{\mu} \bar{\mu}} & =\kappa\left[\frac{\left(x^{\bar{\mu}}\right)^{2}}{r^{2}}\left(\partial_{r} \phi\right)^{2}+\frac{\mu^{2} \phi^{2}}{d+2}\right], \\
\mathfrak{S}_{\bar{\mu} \bar{\nu}} & =\kappa\left[\frac{x^{\bar{\mu}} x^{\bar{\nu}}}{r^{2}}\left(\partial_{r} \phi\right)^{2}\right], \quad \bar{\mu} \neq \bar{v}, \\
\mathfrak{S}_{m n} & =\hat{g}_{m n} \mathfrak{S}_{00}=-\kappa \frac{\mu^{2} \phi^{2}}{d+2} \hat{g}_{m n} .
\end{aligned}
$$

\section{Metric coefficients}

Combining (2.20)-(2.23) with (2.24)-(2.27), we get the system of equations for $\mathfrak{h}_{M N}$ (for the moment we set the constants $\mathcal{C}=\kappa=1$ to simplify the formulas):

$$
\begin{aligned}
& \triangle_{3} \mathfrak{h}_{00}=-\frac{2 \mu^{2} \phi^{2}}{d+2}, \quad \triangle_{3} \xi_{f}=-\frac{2 \mu^{2} \phi^{2}}{d+2} \Rightarrow \mathfrak{h}_{00}=\xi_{f} \\
& \triangle_{3} \mathfrak{h}_{\bar{\mu} \bar{\mu}}-2 \partial_{\bar{\mu}}^{2} \mathfrak{h}_{\bar{\mu} \bar{\mu}}-\partial_{\bar{\mu}}^{2}\left(\mathfrak{h}_{00}-\sum_{\bar{\lambda}} \mathfrak{h}_{\bar{\lambda} \bar{\lambda}}+\xi_{f} d\right) \\
& =2 \frac{\left(x^{\bar{\mu}}\right)^{2}}{r^{2}}\left(\partial_{r} \phi\right)^{2}+2 \frac{\mu^{2} \phi^{2}}{d+2}
\end{aligned}
$$

and

$$
\begin{aligned}
& \partial_{\bar{\mu}} \partial_{\bar{\nu}}\left[\mathfrak{h}_{\bar{\nu} \bar{\nu}}+\mathfrak{h}_{\bar{\mu} \bar{\mu}}+\left(\mathfrak{h}_{00}-\sum_{\bar{\lambda}} \mathfrak{h}_{\bar{\lambda} \bar{\lambda}}+\xi_{f} d\right)\right] \\
& =-2 \frac{x^{\bar{\mu}} x^{\bar{\nu}}}{r^{2}}\left(\partial_{r} \phi\right)^{2}, \quad \bar{\mu} \neq \bar{\nu} .
\end{aligned}
$$

We denote the expression in square brackets in the lhs of (3.3) by $f_{\bar{\mu} \bar{\nu}}$. It can easily be seen that $f_{\bar{\mu} \bar{\nu}}$ is a function of $r$ only. Then we can rewrite the lhs as follows:

$\partial_{\bar{\mu}} \partial_{\bar{\nu}} f_{\bar{\mu} \bar{\nu}}(r)=\frac{x^{\bar{\mu}} x^{\bar{\nu}}}{r^{2}}\left[\partial_{r}^{2} f_{\bar{\mu} \bar{\nu}}-\frac{1}{r} \partial_{r} f_{\bar{\mu} \bar{\nu}}\right], \quad \bar{\mu} \neq \bar{v}$,

where we have used the evident relations of the form $\partial_{\bar{\mu}} f(r)=(\mathrm{d} f / \mathrm{d} r) x^{\bar{\mu}} / r$. Hence, (3.3) results in

$\partial_{r}^{2} f_{\bar{\mu} \bar{\nu}}-\frac{1}{r} \partial_{r} f_{\bar{\mu} \bar{\nu}}=-2\left(\partial_{r} \phi\right)^{2}, \quad \forall(\bar{\mu} \neq \bar{v})$.

From (3.5) we conclude that all $f_{\bar{\mu} \bar{\nu}}$ are equal. This is possible only provided that $\mathfrak{h}_{11}=\mathfrak{h}_{22}=\mathfrak{h}_{33}$, as follows directly from the definition of $f_{\bar{\mu} \bar{\nu}}$. Therefore, after summing over $\bar{\mu}$ in (3.2) and taking into account Eq. (3.1) for $\mathfrak{h}_{00}$ we obtain

$$
\begin{aligned}
\triangle_{3} \mathfrak{h}_{11} & =\frac{1}{2}\left(\partial_{r} \phi\right)^{2}-\frac{1}{2} \frac{d-2}{d+2} \mu^{2} \phi^{2} \\
& =\frac{1}{2}\left[\frac{(\mu r+1)^{2}}{r^{2}}-\frac{d-2}{d+2} \mu^{2}\right] \frac{e^{-2 \mu r}}{r^{2}} .
\end{aligned}
$$

The general solution of this equation has the form

$$
\begin{aligned}
\mathfrak{h}_{11}= & C_{1}+\frac{C_{2}}{r} \frac{d}{2(d+2)} \frac{\mu}{r} e^{-2 \mu r} \\
& -+\frac{e^{-2 \mu r}}{4 r^{2}}-\frac{d}{d+2} \mu^{2} \operatorname{Ei}(-2 \mu r),
\end{aligned}
$$

where $\operatorname{Ei}(x)$ is the exponential integral (see [17], chapter 37). The natural boundary condition $\lim _{r \rightarrow \infty} \mathfrak{h}_{11}(r)=0$ implies $C_{1}=0$. Because there is no delta-like source in the rhs of (3.6), $C_{2}$ is also zero. 
Next, Eqs. (3.1), rewritten as

$\triangle_{3} \mathfrak{h}_{00}=\triangle_{3} \xi_{f}=-\frac{2 \mu^{2}}{d+2} \frac{e^{-2 \mu r}}{r^{2}}$,

under the same boundary conditions as for $\mathfrak{h}_{11}$, admit the solution

$\mathfrak{h}_{00}=\xi_{f}=-\frac{1}{d+2} \frac{\mu}{r} e^{-2 \mu r}-\frac{2}{d+2} \mu^{2} \operatorname{Ei}(-2 \mu r)$.

To restore $\kappa \neq 1$ and $\mathcal{C} \neq 1$, we should multiply the righthand sides of (3.7) and (3.9) by the combination $\kappa \mathcal{C}^{2}$ which has the dimension of length ${ }^{2}$. It can easily be verified that these metric coefficients together with the expressions (2.4) for $\phi$ satisfy Eq. (3.5). Taking into account Eq. (2.19), we obtain also the expression for $\mathfrak{h}_{m n}$ :

$\mathfrak{h}_{m n}=\xi_{f} \hat{g}_{m n}$

The solutions for $\mathrm{h}_{M N}$ were already obtained in [2] (see Eqs. (11) and (12)):

$$
\begin{aligned}
\mathrm{h}_{00} & =-\frac{2}{c^{2}} \frac{S_{D} G_{(D+1)}}{2 \pi \hat{V}_{d}} \frac{1+(1+\Omega) d}{d+2} \frac{m}{r}, \\
\mathrm{~h}_{\bar{\mu} \bar{v}} & =\frac{1-\Omega d}{1+(\Omega+1) d} \mathrm{~h}_{00} \delta_{\bar{\mu} \bar{v}}, \\
\xi_{m} & =-\frac{2 \Omega+1}{1+(\Omega+1) d} \mathrm{~h}_{00}, \Rightarrow \mathrm{h}_{m n}=\xi_{m} \hat{g}_{m n} .
\end{aligned}
$$

Finally, the total perturbations of metric coefficients are given by formulas (2.15) and (2.19).

\section{PPN parameter $\gamma$}

It is well known that $h_{00}$ is related to the gravitational potential: $h_{00}=2 \varphi / c^{2}$ [4]. As directly follows from (3.11) and (3.12), the functions $\mathrm{h}_{00}, \mathrm{~h}_{\bar{\mu} \bar{\mu}} \sim 1 / r$ represent the pure Newtonian contributions to the corresponding metric coefficients $g_{00}$ and $g_{\bar{\mu} \bar{\mu}}$, and the requirement that $\mathrm{h}_{00}=2 \varphi_{N} / c^{2}=$ $-\left(2 / c^{2}\right) G_{N} m / r$ leads to a connection between the Newtonian $G_{N}$ and multidimensional $G_{(D+1)}$ gravitational constants in the absence of the scalar field [3]:

$G_{N}=\frac{S_{D} G_{(D+1)}}{2 \pi \hat{V}_{d}} \frac{1+(1+\Omega) d}{d+2}$.

Additionally, if the scalar field is absent, the ratio

$\gamma=\frac{\mathbf{h}_{\bar{\mu} \bar{\mu}}}{\mathbf{h}_{00}}$

defines the parameterized post-Newtonian (PPN) parameter $\gamma$. In the case of Eqs. (3.11) and (3.12) we obtain
$\gamma=\frac{1-\Omega d}{1+(\Omega+1) d}$.

According to the experimental data of astronomical observations in the solar system, $\gamma$ must be very close to 1 [6-8]. In general relativity, the considered ratio is exactly equal to unity: $\gamma=1$. For Eq. (4.3), we can achieve this value only in the case of black string/brane $\Omega=-1 / 2$ in agreement with $[2,13,14]$. That is, the gravitating masses should have tension in the internal space. However, we do not know a physical reason for a gravitating body to have tension. The dust-like value $\Omega=0$ looks much more reasonable. Therefore, a natural question arises whether the presence of a scalar field can provide such functions $\mathfrak{h}_{00}$ and $\mathfrak{h}_{\bar{\mu} \bar{\mu}}$ that they, first, contain contributions demonstrating a pure Newtonian behavior ${ }^{4}$ at astrophysical scales and, second, contribute to the total ratio $h_{\bar{\mu} \bar{\mu}} / h_{00}$ in such a way that this ratio will be close to a unity for $\Omega=0$. Unfortunately, the answer is negative. To demonstrate it, we consider two limiting cases.

First, we consider the case of a "heavy" scalar field mass $\mu \gg r^{-1}$. Taking into account the asymptotic behavior of the exponential integral $\operatorname{Ei}(x) \rightarrow \exp (x) / x$ when $|x| \rightarrow \infty$ (see [17], chapter 37), we can conclude that in the limit $\mu r \gg 1$ the terms $\mathfrak{h}_{00}, \mathfrak{h}_{11}$ decay much faster (i.e. exponentially) with distance than $\mathrm{h}_{00}, \mathrm{~h}_{11}$. Then at distances $r \gg \mu^{-1}$ the metric coefficients $h_{00} \approx \mathrm{h}_{00}$ and $h_{\bar{\mu} \bar{\mu}} \approx \mathrm{h}_{\bar{\mu} \bar{\mu}}$, and for PPN parameter $\gamma$ we obtain Eq. (4.3) which requires the value $\Omega=-1 / 2$ to have the same accuracy as the general relativity. The relativistic gravitational tests (e.g. the deflection of light and the time delay of radar echoes) take place at distances $r \gtrsim R_{\odot}$ where $R_{\odot}$ is the radius of the Sun. Therefore, at such distances scalar fields with masses $\mu \gtrsim 5.05 \times 10^{-49} \mathrm{~g}$ $\sim 2.83 \times 10^{-16} \mathrm{eV}$ do not affect these gravitational tests.

Next, we consider the case of ultralight scalar field mass $\mu \ll r^{-1}$. Since the exponential integral asymptotically behaves as $\operatorname{Ei}(x) \approx C_{E}+\ln (|x|)+x,|x| \ll 1$ (here, $C_{E}$ stands for Euler's constant) [17], then in the limit $\mu r \ll 1$ we get

$$
\begin{aligned}
\mathfrak{h}_{00} \approx & -\kappa \mathcal{C}^{2} \frac{\mu r}{(d+2) r^{2}}\left[1+2 C_{E} \mu r-4(\mu r)^{2}\right. \\
& +2 \mu r \ln (2 \mu r)] \approx-\frac{\kappa \mathcal{C}^{2}}{d+2} \frac{\mu}{r}, \\
\mathfrak{h}_{\bar{\mu} \bar{\mu}} \approx & \kappa \mathcal{C}^{2} \frac{1}{r^{2}}\left(\frac{1}{4}-\frac{2}{2(d+2)} \mu r\right) \approx \frac{\kappa \mathcal{C}^{2}}{4 r^{2}},
\end{aligned}
$$

\footnotetext{
${ }^{4}$ In the PPN formalism, a static spherically symmetric line element in isotropic coordinates is parameterized in such a way that $h_{00}=-r_{g} / r$ and $h_{\bar{\mu} \bar{\mu}}=-\gamma r_{g} / r$, where $r_{g}=2 G_{N} m / c^{2}$ [5]. In the relativistic gravitational tests, $\gamma$ was estimated for this parametrization. Therefore, to compare with astronomical limitations, we must find such a range of parameters where metric coefficients $\mathfrak{h}_{00}$ and $\mathfrak{h}_{\bar{\mu}} \bar{\mu}$ behave as $1 / r$.
} 
where we have restored the dimensional prefactor $\kappa \mathcal{C}^{2}$. For the total metric coefficients we obtain

$h_{00}=\mathrm{h}_{00}+\mathfrak{h}_{00} \approx-\frac{\kappa c^{2}}{2 \pi \hat{V}_{d}} \frac{1+(1+\Omega) d}{d+2} \frac{m}{r}-\frac{\kappa \mathcal{C}^{2}}{d+2} \frac{\mu}{r}$

and

$h_{\bar{\mu} \bar{\mu}}=\mathrm{h}_{\bar{\mu} \bar{\mu}}+\mathfrak{h}_{\bar{\mu} \bar{\mu}} \approx-\frac{\kappa c^{2}}{2 \pi \hat{V}_{d}} \frac{1-\Omega d}{d+2} \frac{m}{r}+\frac{\kappa \mathcal{C}^{2}}{4 r^{2}}$.

As we mentioned above, metric coefficients should demonstrate the Newtonian $1 / r$ behavior. This means that in the expression (4.7) for $h_{\bar{\mu} \bar{\mu}}$ the second term with $1 / r^{2}$ should be much less than the first one with $1 / r$. Then we get the additional condition for $r$ :

$r \gg \frac{\pi(d+2)}{2} \frac{\mathcal{C}^{2} \hat{V}_{d}}{m c^{2}(1-\Omega d)}$.

Therefore, $h_{\bar{\mu} \bar{\mu}} \approx \mathrm{h}_{\bar{\mu} \bar{\mu}}$.

To be in agreement with the relativistic gravitational tests (i.e. to have $\gamma \approx 1$ ), we must equate $h_{00}$ and $h_{\bar{\mu} \bar{\mu}}$. This results in the relation:

$-d(1+2 \Omega) m c^{2}=2 \pi \hat{V}_{d} \mathcal{C}^{2} \mu$.

This equation shows that the mass of scalar field $\mu$ is defined by the mass of the gravitating body. Since the scalar field and the gravitating body are independent, such condition looks very artificial. Moreover, the condition of positivity ${ }^{5}$ of $\mu$ : $\Omega<-1 / 2$ excludes the sought value $\Omega=0$. Therefore, we can satisfy (4.9) only if both the lhs and the rhs are equal to zero simultaneously. Obviously, this is possible either when $\mu=0$ (massless field), or when $\mathcal{C}=0$ (absence of the field at all), but in both cases $\Omega=-1 / 2$. It follows from the above that a massless scalar field does not affect the gravitational test for the PPN parameter $\gamma$ in the solar system subject to the condition (4.8), where $r$ is replaced by $R_{\odot}$ and $m$ by $M_{\odot}$.

Therefore, the presence of the scalar field (massive or massless) does not improve the situation with respect to the parameter $\Omega$. In all cases, we arrive at the same conclusion that to be in agreement with the relativistic gravitational tests the gravitating mass should have tension: $\Omega=-1 / 2$.

\section{Conclusion}

In this paper, we have considered the effect of a scalar field on the PPN parameter $\gamma$ in a multidimensional Kaluza-Klein

\footnotetext{
${ }^{5}$ It is worth noting that in the exotic case of negative mass $\mu<0$, the value $\Omega=0$ is possible.
}

model with Ricci-flat internal space. In this model, the gravitational field is created by two coupled sources. First, this is a point-like massive body which has a dust-like equation of state in the external space and an arbitrary parameter $\Omega$ of the equation of state in the internal space. In the static limit and within the adopted accuracy, the massive gravitating body is smeared over the internal space. The second source is a static spherically symmetric massive scalar field centered at the origin where the point-like massive body is situated. We have assumed also that this scalar field is in its ground state in the internal space and does not depend on the extra spatial coordinates. In the linear approximation, we have calculated the perturbed metric coefficients for this model. We have used these perturbations to calculate the parameterized post-Newtonian parameter $\gamma$. This PPN parameter is well constrained by the gravitational tests (e.g. the deflection of light and the time delay of radar echoes) in the solar system. According to these tests, $\gamma$ is extremely close to unity, which is in very good agreement with the general relativity prediction [6-8]. In Kaluza-Klein models with Ricci-flat internal space, the same value of $\gamma$ as in the general relativity (i.e. $\gamma=1$ ), can be achieve only for black brane value $\Omega=-1 / 2$ [1-3]. However, we consider this value as not very realistic since there is no any physically reasonable motivation for it up to now. Therefore, in the present paper we have investigated a possibility with the help of scalar field to shift $\Omega$ to a more natural value $\Omega=0$. Simultaneously, we have tried to keep the PPN parameter $\gamma$ close to unity. We have shown that we can fulfill the latter condition for massive as well as massless ${ }^{6}$ scalar field. For example, in the solar system to have $\gamma \approx 1$, the mass of scalar field should be $\mu \gtrsim 5.05 \times 10^{-49} \mathrm{~g} \sim 2.83 \times 10^{-16} \mathrm{eV}$. Unfortunately, in all cases, we have arrived at the same conclusion that to be in agreement with the relativistic gravitational tests, the gravitating mass should have tension: $\Omega=-1 / 2$.

Acknowledgements We would like to thank Maxim Eingorn for his contribution during the initial phase of work as well as for stimulating discussions and valuable comments.

Open Access This article is distributed under the terms of the Creative Commons Attribution 4.0 International License (http://creativecomm ons.org/licenses/by/4.0/), which permits unrestricted use, distribution, and reproduction in any medium, provided you give appropriate credit to the original author(s) and the source, provide a link to the Creative Commons license, and indicate if changes were made. Funded by SCOAP ${ }^{3}$.

\footnotetext{
${ }^{6}$ It is well known that massless fields can result in a potentially dangerous situation if they are coupled to matter. This usually leads to the fifth-force problem. We can see it e.g. from Eq. (4.6) obtained in the case of light mass scalar field. Here, we get the additional contribution of the order of $1 / \mathrm{r}$ to the gravitational potential. With respect to the PPN parameter $\gamma$, we eliminated this danger by putting $\mu=0$ and $\Omega=-1 / 2$. However, such fifth force can manifest itself in other experiments. This can result in additional restrictions on the constant of interaction $\mathcal{C}$.
} 


\section{References}

1. M. Eingorn, A. Zhuk, Classical tests of multidimensional gravity: negative result. Class. Quantum Gravity 27, 205014 (2010). arXiv:1003.5690 [gr-qc]

2. A. Chopovsky, M. Eingorn, A. Zhuk, Kaluza-Klein multidimensional models with Ricci-flat internal spaces: the absence of the KK particles. Adv. High Energy Phys. 106135 (2013). arXiv:1311.0220 [gr-qc]

3. A. Chopovsky, M. Eingorn, A. Zhuk, Many-body problem in Kaluza-Klein models with toroidal compactification. Eur. Phys. J. C 74, 2700 (2014). arXiv:1302.0501 [gr-qc]

4. L.D. Landau, E.M. Lifshitz, The Classical Theory of Fields, Course of Theoretical Physics Series, vol. 2, 4th edn. (Pergamon Press, Oxford, 2000)

5. C.M. Will, Theory and Experiment in Gravitational Physics (Cambridge University Press, Cambridge, 2000)

6. B. Bertotti, L. Iess, P. Tortora, A test of general relativity using radio links with the Cassini spacecraft. Nature 425, 374 (2003)

7. S.S. Shapiro, J.L. Davis, D.E. Lebach, J.S. Gregory, Measurement of the solar gravitational deflection of radio waves using geodetic very-long-baseline interferometry data, 1979-1999. Phys. Rev. Lett. 92, 121101 (2004)

8. C.M. Will, Was Einstein right? Testing relativity at the century, in 100 Years of Relativity: Spacetime Structure-Einstein and Beyond, ed. by A. Ashtekar (World Scientific, Singapore, 2005), p. 205. arXiv:gr-qc/0504086
9. J. Traschen, D. Fox, Tension perturbations of black brane spacetimes. Class. Quantum Gravity 21, 289 (2004). arXiv:gr-qc/0103106

10. P.K. Townsend, M. Zamaklar, The first law of black brane mechanics. Class. Quantum Gravity 18, 5269 (2001). arXiv:hep-th/0107228

11. T. Harmark, N.A. Obers, General definition of gravitational tension. JHEP 0405, 043 (2004). arXiv:hep-th/0403103

12. D. Kastor, J. Traschen, Stresses and strains in the first law for Kaluza-Klein black holes. JHEP 0609, 022-039 (2006). arXiv:hep-th/0607051

13. M. Eingorn, A. Zhuk, Kaluza-Klein models: can we construct a viable example? Phys. Rev. D 83, 044005 (2011). arXiv: 1010.5740 [gr-qc]

14. M. Eingorn, O. de Medeiros, L. Crispino, A. Zhuk, Latent solitons, black strings, black branes, and equations of state in Kaluza-Klein models. Phys. Rev. D 84, 024031 (2011). arXiv:1101.3910 [gr-qc]

15. M. Eingorn, A. Zhuk, Remarks on gravitational interaction in Kaluza-Klein models. Phys. Lett. B 713, 154 (2012). arXiv:1201.1756 [gr-qc]

16. A. Chopovsky, M. Eingorn, A. Zhuk, Problematic aspects of Kaluza-Klein excitations in multidimensional models with Einstein internal spaces. Phys. Lett. B 736, 329 (2014). arXiv: 1402.1340 [gr-qc]

17. K. Oldham, J. Myland, J. Spanier, An Atlas of Functions, with Equator, The Atlas Function Calculator, 2nd edn. (Springer, Berlin, 2009) 